Toward smarter BCls: extending BCls through hybridization and intelligent control

This article has been downloaded from IOPscience. Please scroll down to see the full text article.

2012 J. Neural Eng. 9013001

(http://iopscience.iop.org/1741-2552/9/1/013001)

View the table of contents for this issue, or go to the journal homepage for more

Download details:

IP Address: 128.178.22.183

The article was downloaded on 06/02/2012 at 10:11

Please note that terms and conditions apply. 


\title{
Toward smarter BCIs: extending BCIs through hybridization and intelligent control
}

\author{
B Z Allison ${ }^{1}$, R Leeb ${ }^{2}$, C Brunner ${ }^{1}$, G R Müller-Putz ${ }^{1}$, G Bauernfeind ${ }^{1}$, \\ J W Kelly ${ }^{3}$ and C Neuper ${ }^{1,4}$ \\ ${ }^{1}$ Institute for Knowledge Discovery, BCI Lab, Graz University of Technology, Krenngasse 37, \\ 8010 Graz, Austria \\ ${ }^{2}$ Chair on Non-Invasive Brain-Machine Interface, Center for Neuroprosthetics, School of Engineering, \\ École Polytechnique Fédérale de Lausanne, Station 11, 1015 Lausanne, Switzerland \\ ${ }^{3}$ Department of Electrical and Computer Engineering, Carnegie Mellon University, Pittsburgh, PA, USA \\ ${ }^{4}$ Department of Psychology, University of Graz, Universitätsplatz 2/III, 8010 Graz, Austria \\ E-mail: allison@tugraz.at
}

Received 22 August 2011

Accepted for publication 14 October 2011

Published 7 December 2011

Online at stacks.iop.org/JNE/9/013001

\begin{abstract}
This paper summarizes two novel ways to extend brain-computer interface (BCI) systems. One way involves hybrid BCIs. A hybrid BCI is a system that combines a BCI with another device to help people send information. Different types of hybrid BCIs are discussed, along with challenges and issues. BCIs are also being extended through intelligent systems.

Software that allows high-level control, incorporates context and the environment and/or uses virtual reality can substantially improve BCI systems. Throughout the paper, we critically address the real benefits of these improvements relative to existing technology and practices. We also present new challenges that are likely to emerge as these novel BCI directions become more widespread.
\end{abstract}

\section{Introduction and scope}

Until a few years ago, most brain-computer interface (BCI) systems only accepted input from one type of electroencephalogram (EEG) signal (such as P300 or steadystate visual evoked potential (SSVEP)), and only allowed simple and inflexible messages or commands (such as spelling or moving a wheelchair forward). Recently, many articles have presented two different ways to make BCI systems more intelligent. First, BCIs can be combined with another communication device. These hybrid BCIs accept input from different signals and/or signal combinations. Second, BCIs can be combined with more intelligent, context aware software that helps users execute desired goals in changing realworld environments.

\section{Hybrid BCIs}

\subsection{Introducing the hybrid BCI}

Recently, several groups have validated a novel approach toward BCI design called a hybrid BCI [1]. After a brief review of different definitions, we recognized that there are some inconsistencies among published BCI definitions, and we agreed on the following definitions of a hybrid BCI and some relevant terms. A hybrid BCI is a BCI combined with at least one other system or device to help people send information. This second communication system or device might be

(i) another BCI (called a pure hybrid);

(ii) a device based on other physiological signals (called a physiological hybrid), such as the electromyogram (EMG, associated with muscle movements), electrooculogram (EOG, associated with eye movements) or heart rate; or

(iii) another communication device (called a mixed hybrid), which could be an assistive technology meant for disabled users or a conventional input such as a keyboard or mouse.

A hybrid BCI can work simultaneously and/or sequentially. In a simultaneous hybrid, the user can operate the BCI and other system(s) at the same time. In a sequential (or interleaved) hybrid, the user might instead choose to engage one of the communication systems only part of the time, 
such as when the user is (or is not) fatigued, or to reduce annoyance and/or improve usability. The secondary system could supplement the primary system in many ways, such as turning it on or off, confirming selections, correcting errors, facilitating immersion, increasing bandwidth, improving training, modifying commands or providing communication options better suited to specific tasks or applications.

\subsection{New work with hybrid BCIs}

Physiological hybrid BCIs were introduced fairly early, but have also been explored recently. Kennedy and colleagues developed a cone electrode that was used in an early hybrid BCI that combined EMG with invasively recorded brain signals [2]. Combinations of EEG and EMG signals for control applications were presented in Junker et al [3], Nelson et al [4, 5] and Leeb et al [6]. Scherer et al used the heart rate to switch a BCI system on and off [7]. Danóczy et al presented a grasping robot arm controlled by gaze and asynchronous EEG BCI [8]. Asynchronous BCIs, unlike synchronous BCIs, allow users to send information at their own pace, without following cues or prompts from the system. Zander et al combined gaze-based input with an asynchronous ERD (event-related desynchronization) based BCI for solving the Midas Touch problem $[9,10]$ of a touchless human-computer interaction (HCI) [11]. The Midas Touch problem occurs when a BCI sends a message or command when the user did not intend to communicate at all. Finally, Usakli et al introduced a hybrid spelling platform based on EOG and EEG measurements [12].

While some early work presented the idea of pure hybrid BCIs [13, 14], they have only recently been validated [1, 15-25]. Hybrid systems that combine BCIs and more mainstream interfaces have not been well explored, but this is likely to change in the near future. Some European and other research efforts (e.g. the EU FP7 TOBI project ${ }^{5}$ ) explicitly aim to combine BCIs with conventional interfaces (such as keyboards, mice or joysticks) and assistive technologies (ATs) (such as systems based on limited residual movement) [26].

Thus, a hybrid BCI is not a specific type of novel BCI, but an entirely new approach to thinking about BCIs. Until recently, BCIs were typically viewed as stand-alone communication systems. Developing a hybrid BCI requires more effort than a conventional simple BCI, and some researchers considered a hybrid BCI impossible because it would be too difficult to focus on multiple tasks.

\subsection{Emerging challenges}

Indeed, a major issue with any hybrid BCI is the seamless integration of different input mechanisms and mental tasks. A $\mathrm{BCI}$ based on visual attention may be hard to integrate with other tasks or interfaces that require visual attention. However, BCIs that combine visual attention and imagined movement are possible $[15,16]$. A related challenge is ensuring that the two interfaces complement each other well. Two different interfaces that both allow users to move the same cursor in the same direction at the same time would seem redundant.

\footnotetext{
5 http://www.tobi-project.org/
}

The secondary system should compensate for some weakness in the principal interface. For example, an SSVEP BCI was recently developed to control an orthosis. Results were promising except for one weakness: a high false positive rate [17]. Therefore, a type of ERD BCI with a very low false positive rate was introduced as a switch to toggle the SSVEP BCI on and off [17]. Similar work validated a hybrid BCI in which users could instead turn an SSVEP BCI on and off using a near-infrared spectroscopy BCI [1].

Another challenge is feedback. In a hybrid BCI, users may need feedback that reflects the efficacy of different input methods. For example, if users try to move a cursor left by simultaneously imagining left hand movement and focusing on a left LED, how can they know whether imagined movement (ERD) or visual attention (SSVEP) played a larger role in the success or failure of the cursor movement [18]? Users might need different feedback bars to present the strength of different input signals. Alternately, the BCI might be designed so that the feedback is more intuitive. For example, if the user moves in one dimension with SSVEP activity, and another dimension with ERD, then the subject could easily adjust mental strategies to improve control as needed [25]. Feedback may be easier with interleaved hybrid BCIs, in which each interface controls different functions or is only active at certain times [22, 27].

A hybrid BCI could also include feedback from the user to the BCI. Error potentials (ErrPs) occur shortly after a user realizes that he or she made an error [28], and ErrPs could provide a feedback signal to improve the effectiveness of BCIs [29-31]. Later research showed that ErrPs occur when a user realizes that the BCI made a mistake, and that ErrPs can be detected in single trials $[32,33]$. ErrPs could be used as part of a hybrid BCI system in a few ways. ErrPs could prevent the BCI from implementing a command, reverse or modify a previously sent command, inform a listener or device that an error occurred or contribute to a reinforcement learning mechanism to improve the BCI's classifier. ErrP activity could also be combined with conventional interfaces. While users can convey that an error was made by hitting the backspace key or back button, ErrP activity could provide a signal more quickly and unobtrusively [34].

\subsection{Making hybrid BCIs practical-bringing BCIs to the patient}

While all of these challenges are important, getting hybrid (and other) BCIs to the people who need them is always crucial as well. Despite the considerable progress in BCI research recently, and the availability of many improved BCI systems, their communication and control capabilities are still very limited compared to natural communication or existing ATs. Practical brain-computer interfaces for disabled people should allow them to use all their remaining abilities as control possibilities. Sometimes users have residual muscle activity, typically in the morning when they are not exhausted. With a hybrid BCI, patients could use this muscular activity when possible and switch to a BCI when fatigued. In such a hybrid BCI, conventional ATs (operated using some residual muscular functionality) are enhanced by BCI technology. 
Millán et al reviewed the state of the art and challenges in combining BCI and AT [27]. This paper identified the following directions and issues for a new generation of hybrid BCI assistive technology for people with physical disabilities.

(i) Conventional AT will be enhanced by BCI technology: by adding an additional degree of freedom, by enhancing the robustness of the control signals (combining EEG and other AT) or by improved interaction. BCIs will expand the range of opportunities available to AT teams worldwide for building flexible and personalized solutions for their clients' needs.

(ii) Equipping BCI-assistive devices with novel self-adaptive capabilities: e.g. by incorporating fusion techniques for the combination of EEG with other signals, automatic selection of EEG phenomena, on-line adaptation to EEG changes, using modern HCI principles for shaping the interaction and recognizing mental states and cognitive processes.

(iii) $\mathrm{BCI}$ will become more robust: combining EEG with other signals will allow users to become more autonomous and interact over long periods of time.

(iv) BCI will increase its performance and reliability significantly: modern HCI and shared autonomy principles will make it possible.

(v) BCI will reduce cognitive effort: this will be possible because of modern HCI and the recognition of the user's mental states and cognitive processes.

(vi) BCI will be easier: the design of efficient training protocols will accelerate, improve and make more intuitive each user's mastering of the BCI assistive technology; also, the development of new electrodes and aesthetic helmets will facilitate operation of BCI by laypeople.

(vii) Novel BCI designs will ensure that outcomes follow standard BCI assistive technology: lack of coordination in BCI research has thus far impeded the creation of a shared model and standards among BCI groups.

\section{Shared control and intelligent tools}

Current BCI systems are unreliable, and may not perform well even under optimal conditions. Modern BCIs are characterized by noisy and low bit rate outputs and by the limited range of possible commands. Early BCIs simply acted on a subject's brain activity regardless of the context. More recent work developed more intelligent systems by introducing high level (or goal-oriented) behavior, incorporating context, such as the state of the system, surrounding environment or mental state of the user.

\subsection{Goal versus process control}

Since (at least) non-invasive BCIs are low-bandwidth systems, users who must painstakingly communicate all details of messages or commands may need a long time. Specifying the details of hand movements needed to get a glass of water, or the individual movements to navigate through a house, would be impractical. In a goal-oriented approach, the user produces high-level commands or intentions, but is freed from the burden of the low-level and demanding highspeed interactions. Two interacting agents, the BCI system and the intelligent device, are sharing the task. Shared control has been prominent in recent work regarding neuroprostheses such as robots and wheelchairs [35-40], as well as other intelligent systems such as smart virtual keyboards [41] or virtual helicopters [42].

For example, a user who is writing a novel such as 'The Diving Bell and the Butterfly' could write more quickly with intelligent tools that can predict letters, complete words or automatically correct errors. Consider a user who wishes to move a wheelchair, robot or virtual avatar to a new location. Instead of specifying each movement, a user could simply select a destination and leave the rest of the work to the system. This remaining work is not trivial. For example, the system must determine a path to the new location. It must avoid obstacles, which may be new (such as a toy that was just placed in the path of a wheelchair). Thus, it needs a sensing mechanism to detect obstacles, and software to develop and implement new paths accordingly, which must function in realtime.

Shared autonomy frameworks such as these aim to integrate the user's mental commands with the contextual information gathered by the intelligent device (such as a language model based on written characters or obstacles perceived by the robot sensors) to help the user to reach the target/goal more quickly and reliably. Shared control can make goal-oriented control easier, avoid pointless mental commands, and can help determine meaningful motion sequences (e.g. for a neuroprosthesis). Furthermore, shared autonomy will be a key component of future hybrid BCIs, since shared autonomy can help shape the closed-loop dynamics between the user and the brain-actuated device to perform tasks as easily as possible.

An important challenge will be providing users with both high-level and low-level control, as well as an easy way to switch between them. While a system that allows only lowlevel commands could be slow and frustrating, a system that allows only high-level commands could also be problematic. A user who cannot specify a new message or destination would be limited to some preprogrammed high-level commands, and might require technical support to make even minor changes.

Therefore, shared control should keep the user in the loop to avoid becoming a fully autonomous or stand-alone system. For example, if the user indicates with a high-level command to go to a new location, he still can influence the path or change the target location en route. Consider a situation in which the user wants to go to the kitchen to see his wife and selects an icon for 'go to the kitchen' using his BCI. While he is passing by the corridor, he sees her in the bedroom, so he must have some way to intervene and avoid an unnecessary trip. Furthermore, the system should ask the user for new directions or help if he reaches an unknown point where new input is required. Hence, shared control certainly does not refer to a fully autonomous or stand-alone system. Shared control involves close interaction between the system and the user. 
Recent work [43] compared high-level (or goal oriented) control to the activity of the cortex and other brain areas, and low-level (or process oriented) control to the activity of lower nervous systems and muscles. In a healthy person, goals are decided by the brain, and most details of the execution are mediated unconsciously by lower areas. People who move from one room to another do not think about all of the muscle activities needed to get there. Similarly, users should have the option of conveying only their goals, while the BCI handles other details.

\subsection{System and environment}

Two closely related issues are the system and environment. A BCI system could use information about its own state in different ways. For example, if a door is open or a light is on, then a smart home system should not present BCI users with these possible commands. Perhaps the icons reflecting these commands could be grayed out. This intelligent control can also be used to provide inference-based technology to further help users. If a user launches a movie with his BCI, the system might infer that the user would like the room dark, and will turn off any lights. If a user activates a security camera or tries to move toward a room, the BCI system might activate lights in that room. 'Ambient intelligence' could also involve intelligent personalization so that inferences are unique to each person's preferences $[44]^{6}$.

BCIs can incorporate information about the environment as well. In Millán et al [45], a mobile robot moved according to both the user's mental command and the robot's location relative to obstacles. Thus, if a user thought about a mental task the robot might turn left or follow a wall to the left based on environmental cues such as the presence of a wall to the left. There are various ways that intelligent systems could further utilize environmental information, such as data about external lighting, temperature, sonic or electrical noise (which could affect the tasks involved in BCIs or signal quality), or availability of resources (so a user cannot try to pour juice from an empty juice bottle).

Contextual information could lead to a 'semisynchronous' BCI that overcomes drawbacks of synchronous and asynchronous BCIs. Synchronous BCIs require users to coordinate their mental activities with specific cues. Asynchronous BCIs do not have this drawback; users may communicate whenever they wish. However, because they are always on, asynchronous BCIs risk a high false positive rate, and hence may employ means to confirm selections. In a semi-synchronous BCI, users could send signals whenever they want. The system uses the context to infer whether signals are false positives or really reflect user intent (and perhaps which intent). Consider an example of a BCI-based soccer game. If a user thinks about moving his feet when there is no ball nearby, then he runs. If there is a ball nearby, then the imagined foot movement causes the avatar to run with the ball. If the user is also focusing attention on a virtual teammate, then the same mental activity will cause the avatar to kick the ball to the teammate. If the user thinks about moving his feet

\footnotetext{
6 http://www.brainable.org/
}

during a break in gameplay, then the system assumes it is a false positive.

A similar example is being pursued in the EU FP7 TREMOR project $^{7}$. This project presents a system that can detect imagined movements from brain activity. If the user imagines movement and then tries to move the hand, then the movement occurs normally. However, if no imagined movement precedes a hand movement, then the hand movement is suppressed with a functional electrical stimulation (FES) system. This device could prevent involuntary tremors, which often affect persons who have had a stroke. This is a different perspective toward a 'semisynchronous' BCI. The BCI element of the system is the opposite of a normal BCI: the device only takes action if the user does not think of movement. Still, this is a novel BCI system that combines a BCI with a new type of contextual information (the presence of EMG activity that reflects arm movement).

\subsection{Novel BCI applications using virtual reality techniques}

Because BCIs are closed-loop systems, feedback is an important component of them. Various methods of providing feedback can inform the participant about success or failure of an intended act. Thus, feedback either supports reinforcement during the learning/training process or in controlling the application. In particular, virtual reality (VR) has been proven to be an interesting and promising way to realize such feedback. Several prototypes have enabled users to navigate in virtual scenes solely by means of their oscillatory cerebral activity, recorded on the scalp via EEG electrodes. Healthy participants explored virtual spaces [46-49] and manipulated virtual objects [50]. A spinal-cord injured patient controlled a wheelchair through a virtual street [51]. Additionally, evoked potentials (P300) [52] and SSVEPs [53, 54] have been used to control VR feedback and augmented reality [55]. The BCI users who use immersive virtual environments (VEs) made fewer errors, reported that BCIs were easier to learn and use, and stated that they enjoy BCI use more $[46,48,56]$. These benefits may occur because VEs enhance vividness and mental effort, which may lead to more distinct brain patterns and improve pattern recognition performance. VR technologies provide motivating, safe and controlled conditions that could facilitate both BCI learning and research into the brain responses and neural processes involved.

Figure 1 presents different VR environments controlled by different BCI approaches. The BCI system in panels (a) and (b) allows users to navigate via imagined movement. Users can move forward by imagining foot movement, and can move left or right by imagining left or right hand movement. Panel (c) shows an even newer BCI system in which users navigate through visual attention. The avatar is surrounded by three red boxes, which each oscillate at different frequencies. Users can move left, right, or forward by focusing on the corresponding box.

A more recent $\mathrm{BCI}$ allows users to move in a $\mathrm{VE}$ and also send context-dependent commands. We developed a BCI

\footnotetext{
7 http://www.iai.csic.es/tremor/
} 

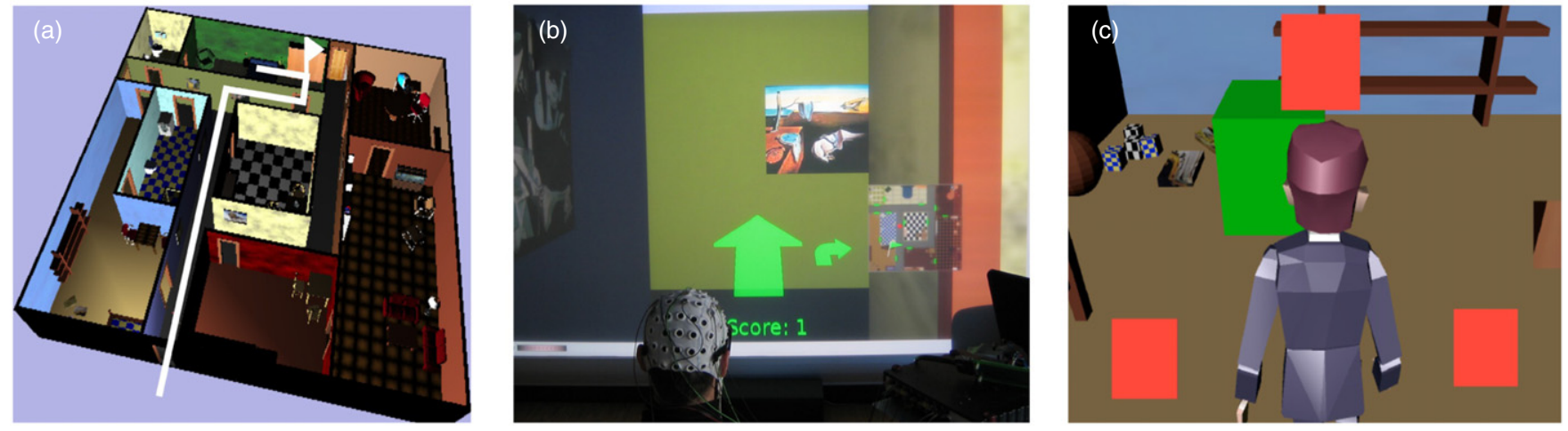

Figure 1. Two different ways to use a BCI to navigate through a virtual apartment in VR. Panel (a) presents an overview of a virtual apartment and (b) shows an image of a subject navigating through that virtual apartment with motor imagery [46]. Panel (c) shows an SSVEP BCI that can allow an avatar to move through an apartment or other environment [53].

(This figure is in colour only in the electronic version)

system that allows users to control the popular online game World of Warcraft based on ERD activity associated with imagination of left hand, right hand or feet movement [57]. In some environments, these commands cause the avatar to rotate left or right or move forward. However, when the avatar is talking to a game-generated character to obtain or redeem a quest, movement is not needed, and so the three commands produce other effects. The context-dependent BCI also allows different commands when the avatar is in combat or collecting loot. Further directions may explore hybridizing this system with SSVEP measures, other brain signals or conventional manual commands.

\section{Summary}

In summary, extending BCIs by combining them with other communication devices and intelligent systems could substantially improve BCIs in many ways. Hybrid BCIs represent a fundamentally different view of the 'input' side of BCIs, allowing users to communicate via whatever signal(s) or combinations work best different times, situations and goals. Similarly, shared control is a new perspective on the 'output' side of BCIs. Users could accomplish goals more quickly and intuitively without unnecessary effort. These developments could make BCI systems more reliable, flexible, usable and powerful.

\section{Acknowledgments}

The research leading to these results has received funding from the European Union Seventh Framework Programme FP7/2007-2013 under grant agreement 248320.

\section{References}

[1] Pfurtscheller G, Allison B Z, Bauernfeind G, Brunner C, Solis Escalante T, Scherer R, Zander T O, Müller-Putz G, Neuper C and Birbaumer N 2010 The hybrid BCI Front. Neurosci. 442
[2] Kennedy P R, Bakay R A, Moore M M, Adams K and Goldwaithe J 2000 Direct control of a computer from the human central nervous system IEEE Trans. Rehabil. Eng. 8 198-202

[3] Junker A, Berg C and Schnider P 1995 Evaluation of the cyberlink interface as an alternative human operator controller Technical Report Wright-Patterson Air Force Base, $\mathrm{OH}$

[4] Nelson T W, Hettinger L J, Cunningham J A, Roe M M, Lu L G, Haas M W, Dennis L B, Pick H L and Junker A 1996 Brain-body actuated control: assessment of an alternative control technology for virtual environments Image Conf.

[5] Nelson W T, Hettinger J, Cunningham J A, Roe M M, Haas M W and Dennis L B 1997 Navigating through virtual flight environments using brain-body-actuated control Virtual Reality Annu. Int. Symp. p 30

[6] Leeb R, Sagha H, Chavarriaga R and Millán J d R 2011 A hybrid brain-computer interface based on the fusion of electroencephalographic and electromyographic activities J. Neural Eng. 8025011

[7] Scherer R, Müller-Putz G R and Pfurtscheller G 2007 Self-initiation of EEG-based brain-computer communication using the heart rate response J. Neural Eng. 4 L23-9

[8] Danoczy M, Fazli S, Grozea C, Müller K R and Popescu F 2008 Brain2robot: a grasping robot arm controlled by gaze and asynchronous EEG BCI Proc. 4th Int. BCI Workshop and Training Course

[9] Jacob R J K, Legett J J, Myers B A and Pausch R 1993 Interaction styles and input/output devices Behav. Inf. Technol. 12 69-79

[10] Moore M M 2003 Real-world applications for brain-computer interface technology IEEE Trans. Neural Syst. Rehabil. Eng. 11 162-5

[11] Zander T O, Gaertner M, Kothe C and Vilimek R 2011 Combining eye gaze input with a brain-computer interface for touchless human-computer interaction Int. $J$. Hum.-Comput. Interact. 27 38-51

[12] Usakli A B, Gurkan S, Aloise F, Vecchiato G and Babiloni F 2009 A hybrid platform based on EOG and EEG signals to restore communication for patients afflicted with progressive motor neuron diseases Conf. Proc. IEEE Engineering in Medicine and Biology Society (Minneapolis, MN, 3-6 September 2009) pp 543-6

[13] Allison B Z, Vankov A and Pineda J A 1999 EEGs and ERPs associated with real and imagined movement of single 
limbs and combinations of limbs and applications to brain-computer interface (BCI) systems Soc. Neurosci. Abstr. 251139

[14] Wolpaw J R, Birbaumer N, McFarland D J, Pfurtscheller G and Vaughan T M 2002 Brain-computer interfaces for communication and control Clin. Neurophysiol. $113767-91$

[15] Allison B Z, Brunner C, Kaiser V, Müller-Putz G R, Neuper C and Pfurtscheller G 2010 Toward a hybrid brain-computer interface based on imagined movement and visual attention J. Neural Eng. 7026007

[16] Brunner C, Allison B Z, Krusienski D J, Kaiser V, Müller-Putz G R, Pfurtscheller G and Neuper C 2010 Improved signal processing approaches in an offline simulation of a hybrid brain-computer interface J. Neurosci. Methods 188 165-73

[17] Pfurtscheller G, Solis-Escalante T, Ortner R, Linortner P and Muller-Putz G R 2010 Self-paced operation of an SSVEP-based orthosis with and without an imagery-based 'brain switch': a feasibility study towards a hybrid BCI IEEE Trans. Neural Syst. Rehabil. Eng. 18 409-14

[18] Brunner C, Allison B Z, Altstätter C and Neuper C 2011 A comparison of three brain-computer interfaces based on event-related desynchronization, steady state visual evoked potentials, or a hybrid approach using both signals J. Neural Eng. 8025010

[19] Jin J, Allison B Z, Wang X and Neuper C 2011 A hybrid brain-computer interface based on P300 and M-VEP 5th Int. BCI Conf. (Graz) pp 224-7

[20] Li Y, Long J, Yu T, Yu Z, Wang C, Zhang H and Guan C 2010 An EEG-based BCI system for 2-D cursor control by combining mu/beta rhythm and P300 potential IEEE Trans. Biomed. Eng. 57 2495-505

[21] Ferrez P W and Millán J del R 2008 Simultaneous real-time detection of motor imagery and error-related potentials for improved BCI accuracy Proc. 4th Int. Brain-Computer Interface Workshop and Training Course (Graz, Austria)

[22] Su Y, Qi Y, Luo J-X, Wu B, Yang F, Li Y, Zhuang Y-T, Zheng $\mathrm{X}-\mathrm{X}$ and Chen W-D 2011 A hybrid brain-computer interface control strategy in a virtual environment J. Zhejiang Univ. Sci. C 12 351-61

[23] Panicker R C, Puthusserypady S and Sun Y 2011 An asynchronous P300 BCI with SSVEP-based control state detection IEEE Trans. Biomed. Eng. 58 1781-8

[24] Jin J, Allison B Z, Wang X and Neuper C 2011 A comparison of three brain-computer interfaces based on P300 potentials, M-VEPs or a hybrid approach using both signals IEEE Trans. Neural Syst. Rehabil. Eng. (in review)

[25] Allison B Z, Brunner C, Grissmann S, Altstätter C, Wagner I and Neuper C 2011 A hybrid ERD/SSVEP BCI for continuous simultaneous two-dimensional cursor control (in review)

[26] Kreilinger A, Kaiser V, Breitwieser C, Williamson J, Neuper C and Müller-Putz G R 2011 Switching between manual control and brain-computer interface using long term and short term quality measures Front. Neuroprosthetics (in revision)

[27] Millán J d R et al 2010 Combining brain-computer interfaces and assistive technologies: state-of-the-art and challenges Front. Neurosci. 4161

[28] Holroyd C B and Coles M G H 2002 The neural basis of human error processing: reinforcement learning, dopamine, and the error-related negativity Psychol. Rev. 109 679-709

[29] Schalk G, Wolpaw J R, McFarland D J and Pfurtscheller G 2000 EEG-based communication: presence of an error potential Clin. Neurophysiol. 111 2138-44

[30] Blankertz B, Dornhege G, Schäfer C, Krepki R, Kohlmorgen J, Müller K R, Kunzmann V, Losch F and Curio G 2003
Boosting bit rates and error detection for the classification of fast-paced motor commands based on single-trial EEG analysis IEEE Trans. Neural Syst. Rehabil. Eng. 11 127-31

[31] Parra L C, Spence C D, Gerson A D and Sajda P 2003 Response error correction-a demonstration of improved human-machine performance using real-time EEG monitoring IEEE Trans. Neural Syst. Rehabil. Eng. 11 173-7

[32] Buttfield A, Ferrez P W and Millán J d R 2006 Towards a robust BCI: error potentials and online learning IEEE Trans. Neural Syst. Rehabil. Eng. 14 164-8

[33] Ferrez P W and Millán J del R 2008 Error-related EEG potentials generated during simulated brain-computer interaction IEEE Trans. Biomed. Eng. 55 923-9

[34] Nijholt A, Tan D, Pfurtscheller G, Brunner C, Millán J del R, Allison B Z, Graimann B, Popescu F, Blankertz B and Müller K-R 2008 Brain-computer interfacing for intelligent systems IEEE Intell. Syst. 23 72-9

[35] Bell C J, Shenoy P, Chalodhorn R and Rao R P N 2008 Control of a humanoid robot by a noninvasive brain-computer interface in humans J. Neural Eng. 5 214-20

[36] Cherubini A, Oriolo G, Macrì F, Aloise F, Cincotti F and Mattia D 2008 A multimode navigation system for an assistive robotics project Auton. Robots 25 383-404

[37] Galán F, Nuttin M, Lew E, Ferrez P W, Vanacker G, Philips J and Millán J Del R 2008 A brain-actuated wheelchair: asynchronous and non-invasive brain-computer interfaces for continuous control of robots Clin. Neurophysiol. 119 2159-69

[38] Graimann B, Allison B Z, Mandel C, Lüth T, Valbuena D and Gräser A 2008 Non-invasive brain-computer interfaces for semi-autonomous assistive devices Robust Intelligent Systems (London: Springer) pp 113-37

[39] Tonin L, Leeb R, Tavella M, Perdikis S and Millán J del R 2010 The role of shared-control in BCI-based telepresence Proc. 2010 IEEE Int. Conf. on Systems, Man and Cybernetics pp 1462-6

[40] Rebsamen B, Guan C, Zhang H, Wang C, Teo C, Ang M H and Burdet E 2010 A brain-controlled wheelchair to navigate in familiar environments IEEE Trans. Neural Syst. Rehabil. Eng. 18 590-8

[41] Williamson J, Murray-Smith R, Blankertz B, Krauledat M and Müller K R 2009 Designing for uncertain, asymmetric control: interaction design for brain-computer interfaces Int. J. Human Comput. Stud. 67 827-41

[42] Royer A S, Doud A J, Rose M L and He B 2010 EEG control of a virtual helicopter in 3-dimensional space using intelligent control strategies IEEE Trans. Neural Syst. Rehabil. Eng. 18 581-9

[43] Wolpaw J R 2007 Brain-computer interfaces as new brain output pathways J. Physiol. $579613-9$

[44] Navarro N A, Ceccaroni L, Velickovski F, Torrellas S, Miralles F, Allison B Z, Scherer R and Faller J 2011 Context awareness as an enhancement of brain-computer interfaces Int. Workshop on Ambient Assisted Living (Malaga, Spain) pp 216-23

[45] Millán J del R, Renkens F, Mouriño J and Gerstner W 2004 Noninvasive brain-actuated control of a mobile robot by human EEG IEEE Trans. Biomed. Eng. 51 1026-33

[46] Leeb R, Lee F, Keinrath C, Scherer R, Bischof H and Pfurtscheller G 2007 Brain-computer communication: motivation, aim and impact of exploring a virtual apartment IEEE Trans. Neural Syst. Rehabil. Eng. 15 473-82

[47] Leeb R, Settgast V, Fellner D W and Pfurtscheller G 2007 Self-paced exploring of the Austrian National Library through thoughts Int. J. Bioelectromagnetism $9237-44$ 
[48] Ron-Angevin R, Diaz-Estrella A and Velasco-Alvarez F 2009 A two-class brain-computer interface to freely navigate through virtual worlds Biomed. Tech. 54 126-33

[49] Scherer R, Lee F, Schlögl A, Leeb R, Bischof H and Pfurtscheller G 2008 Toward self-paced brain-computer communication: navigation through virtual worlds IEEE Trans. Biomed. Eng. 55 675-82

[50] Lecuyer A, Lotte F, Reilly R B, Leeb R, Hirose M and Slater M 2008 Brain-computer interfaces, virtual reality, and videogames Computer 41 66-72

[51] Leeb R, Friedman D, Müller-Putz G R, Scherer R, Slater M and Pfurtscheller G 2007 Self-paced (asynchronous) BCI control of a wheelchair in virtual environments: a case study with a tetraplegic Comput. Intell. Neurosci. 2007 79642

[52] Bayliss J D 2003 Use of the evoked potential P3 component for control in a virtual apartment IEEE Trans. Neural Syst. Rehabil. Eng. 11 113-6

[53] Faller J, Müller-Putz G, Schmalstieg D and Pfurtscheller G 2010 An application framework for controlling an avatar in a desktop-based virtual environment via a software SSVEP brain-computer interface Presence: Teleoperators Virtual Environ. 19 25-34

[54] Lalor E C, Kelly S P, Finucane C, Burke R, Smith R, Reilly R B and McDarby G 2005 Steady-state VEP-based brain-computer interface control in an immersive 3-d gaming environment EURASIP J. Appl. Signal Process. $193156-64$

[55] Faller J, Allison B, Brunner C, Schmalstieg D and Pfurtscheller G 2010 A software SSVEP BCI integrating stimuli within motivating and immersive virtual and augmented reality environments Real Actions in Virtual Environments (RAVE) Conf. (Barcelona, Spain)

[56] Leeb R et al 2006 Walking by thinking: the brainwaves are crucial, not the muscles! Presence: Teleoperators Virtual Environ. 15 500-14

[57] Scherer R, Friedrich E V C, Allison B Z, Pröll M, Chung M, Cheung W, Rao R P N and Neuper C 2011 Non-invasive brain-computer interfaces: enhanced gaming and robotic control Int. Workshop on Artificial Neural Networks (Malaga, Spain) pp 362-7 